\title{
LA-UR-17-26996
}

Approved for public release; distribution is unlimited.

Title: $\quad$ Conducting Field Interactions and Monitored Evolutions

Author(s): $\quad$ Saeger, Kevin J.

Intended for: Training

Issued: 
Disclaimer:

Los Alamos National Laboratory, an affirmative action/equal opportunity employer, is operated by the Los Alamos National Security, LLC for the National Nuclear Security Administration of the U.S. Department of Energy under contract DE-AC52-06NA25396. By approving this article, the publisher recognizes that the U.S. Government retains nonexclusive, royalty-free license to publish or reproduce the published form of this contribution, or to allow others to do so, for U.S. Government purposes. Los Alamos National Laboratory requests that the publisher identify this article as work performed under the auspices of the U.S. Department of Energy. Los Alamos National Laboratory strongly supports academic freedom and a researcher's right to publish; as an institution, however, the Laboratory does not endorse the viewpoint of a publication or guarantee its technical correctness. 


\section{Conducting Field Interactions and Monitored Evolutions}

Online Training

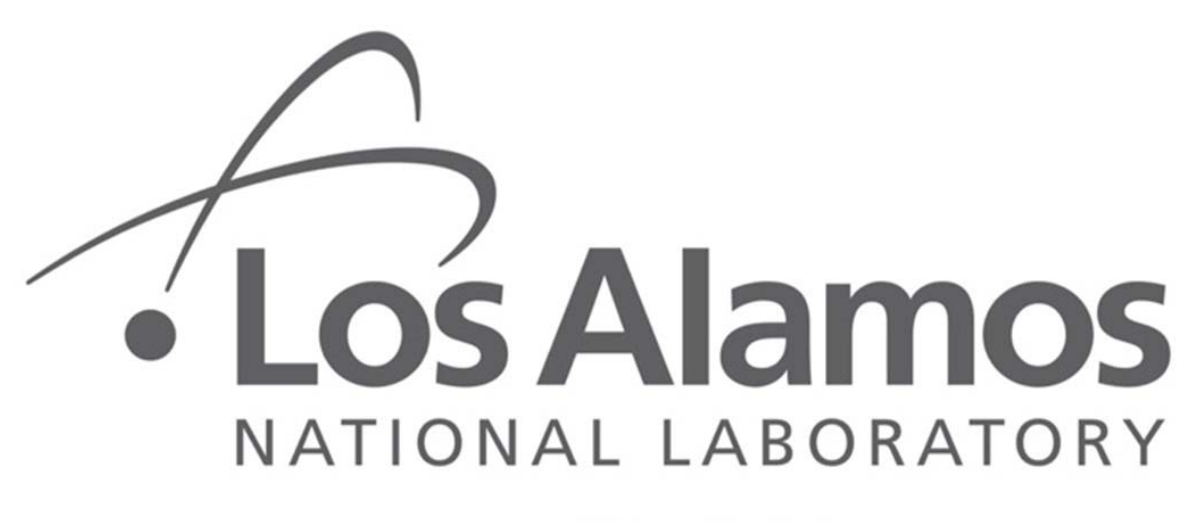

28 July 2017

EST.1943

NNS踹 


\section{Objectives of This Briefing}

- At the end of this training, you will be able to

- Understand the importance of observing and providing feedback on activities

- Conduct Field Interactions, including Monitored Evolutions, as part of the improved Management Observation and Verification (MOV) process

- Understand the importance of the post-interaction dialogue, review, and remediation process 


\section{We Can Achieve Our Full Potential Only by Rigorously Reviewing Everything We Do}

- We strive for unparalleled EXCELLENCE by

- Delivering mission success through operational effectiveness and scientific excellence and

- Inspiring the best talent to perform at the highest level through mission-focused teams

- Managers are essential to achieving organizational excellence by

- Regularly interacting with staff doing work

- Learning about activities, successes, and challenges through interactive dialogues

- Monitoring complete work evolutions to best understand the end-to-end process

- Continuously monitoring, assessing, and improving all the Laboratory's activities are essential to the Laboratory's character: Tenacious, Trusted, Principled, and Scientific 


\section{The Current MOV System Has Been Enhanced to Focus on Improving Research and Operations Activities}

- In practice, the MOV System has provided valuable feedback on the static condition

- Facilities and infrastructure

- Employee well-being

- The MOV System will now provide an additional focus on the dynamic condition

- Field interactions with workers, discussing and performing work, with additional emphasis on maintaining an accurate understanding of work conditions in the field

- Monitored evolutions of activities as they evolve end-to-end. The purpose is to verify that the actual process is evolving as it was specified 


\section{Field Interactions Encourage Learning among the Participants with a Three-Step Process: Questioning, Confirming, and Assessing}

- Questioning-manager seeks input from staff on the efficacy of work documents and conditions in the field

- Confirming-manager communicates with staff during a work evolution and provides confirmation/reinforcement of performance considered to meet expectations, adjusting those expectations as the organization learns

- Assessing-manager observes performance that does not meet expectations, provides assistance in the form of

- Clarified expectations

- Improved work documents

- Additional training

- Other performance resources

The utility of ongoing focused and robust conversations powered by a healthy uneasiness cannot be overstated. 


\section{Monitored Evolutions Verify That Work Is Executed in the Way That It Was Envisioned}

- It is a formal assessment of an evolution for which a checklist or procedure has been established

- It is a scheduled event. Both the monitor and the person being monitored know where, when, and why it is occurring

- It is conducted primarily by first- and second-level line managers 


\section{When Conducting a Monitored Evolution, Following the Steps Is Important to the Effectiveness of the Assessment}

- Schedule the monitored evolution, and review the associated checklist/procedure

- Conduct the monitored evolution

- Watch, listen, and record the monitored worker's actions. Monitor without comment.

- Intervene only if an unsafe or unsecure action is about to occur

- Review results of the Monitored Evolution with the person conducting the evolution

- Compare the record of worker actions to the requirements of the checklist/procedure.

- Document Monitored Evolution findings

- Make improvements to the evolution process when necessary 


\section{Managers Should Be Especially Careful to Look for the Following Actionable Behaviors during a Monitored Evolution}

- Not using the procedure, if required

- Incorrectly performing the procedure

- Using a poorly structured procedure

- Not executing the procedure as written (often resulting in a Stop Work situation)

- Using noncompliant tools, diagnostic equipment, or measurement standards

- Not using prescribed supporting documentation. 


\section{Providing Constructive Feedback Is the Most Valuable Part of the Process and Leads to Vital Learning}

- The monitor promptly reviews findings with the worker

- The monitor reinforces desired behaviors and documents best practices

- The monitor also provides feedback and coaching to the worker to improve performance

- Field Interactions and Monitored Evolutions start a conversation with the worker concerning the efficacy of the evaluated activity 


\section{Document Field Interactions and Monitored Evolutions in the Existing MOV System}

- Ensure that existing MOV Sharepoint Sites have been modified to capture Monitored Evolutions

- Use the "MOV Objective" selection to specify that the Manager and Employee interaction was a "Monitored Evolution" or a "Field Interaction"

- Use the "Supporting Information" field to attach the procedures or checklists that were assessed during the Monitored Evolution

- Use the "MOV Narrative" section to identify any issues associated with the Monitored Evolution or Field Interaction 


\section{Conducting a Monitored Evolution}

\section{Questions?}

Name, phone number, and e-mail of person giving briefing 